\title{
Fuzzy state and output feedback - control for vehicle lateral dynamics
}

\author{
Naoufal El Youssfi ${ }^{{ }_{1}}$, Rachid El Bachtiri ${ }^{1}$, Redouane Chaibi ${ }^{2}$
}

${ }^{1}$ PERE-EST, CED-STI, USMBA Fez, Morocco 2 LESSI-FSDM, CED-STIC, USMBA Fez, Morocco

Email: naoufal.elyoussfi@usmba.ac.ma http://doi.org/10.5339/ qproc.2019.imat3e2018.26

(C) 2019 The Author(s), licensee HBKU Press. This is an open access article distributed under the terms of the Creative Commons Attribution license CC BY 4.0 , which permits unrestricted use, distribution and reproduction in any medium, provided the original work is properly cited.

\section{Abstract}

Research related to the active control systems which improve the stability and the performance of vehicles in critical driving situations has experienced tremendous progress during the last years. Some of the systems have already been installed in passenger cars (ABS, ESP, ACC ...). However, these systems can be further improved using advanced estimation and control design methods [1]. The main goal is still to produce comfortable and safe vehicles. In this work, we study a quadratic stabilization conditions for Takagi-Sugeno (TS) fuzzy control systems, applied to the vehicle lateral dynamics. Stability conditions are represented in the form of LMIs. Hence, we will compare two types of control, to stabilize the dynamics of the vehicle. The first one is the control based on the output feedback, and the second one is the control based on the reconstructed state feedback.

The movement of the vehicle is defined by a set of translations and rotational movements, generally comprised of six main movements; but in this study, we will focus on the vehicle lateral dynamics 2DOF, which can be described by the following equations:

$$
\left\{\begin{array}{l}
m \dot{v}_{y}=m \dot{\psi} v_{y}+2\left(F_{y f}+F_{y r}\right) \\
I_{z} \dot{\psi}=2 a_{r} F_{y r}-2 a_{f} F_{y f}
\end{array}\right.
$$

The difficulty of obtaining a correct vehicle model is that the contact forces are difficult to measure and to model, using the method based on TS models proposed in [2], which are a very interesting mathematical representation of nonlinear systems, because they allow representing any nonlinear system, by a simple structure based on linear models.

The characteristics of the tires are generally assumed that the rear and front lateral forces are modeled with the magic formula as given in [3]. The overall forces are obtained by:

$$
\left\{\begin{array}{l}
F_{y f}=\sum_{i=1}^{2} \mu\left(\left|\alpha_{f}\right|\right) C_{f i} \alpha_{f}(t) \\
F_{y r}=\sum_{i=1}^{2} \mu\left(\left|\alpha_{f}\right|\right) C_{r i} \alpha_{r}(t)
\end{array}\right.
$$

where $C_{f}, C_{r i}$ are the front and rear tire cornering stiffness, respectively, which depend on the road friction coefficient and the vehicle parameters.

Using (2) to approximate the lateral cornering forces, the T-S model can be written in the following form:

$$
\left\{\begin{array}{l}
\dot{x}(\mathrm{t})=\sum_{i=1}^{2} \mu\left(\left|\alpha_{f}\right|\right)\left[A_{i} x(t)+B_{i} u(t)\right] \\
y(\mathrm{t})=\sum_{i=1}^{2} \mu\left(\left|\alpha_{f}\right|\right)\left[C_{i} x(t)+D_{i} u(t)\right]
\end{array}\right.
$$

As a conclusion, in this study, two types of stabilization conditions for vehicle lateral dynamics are presented. The first is for fuzzy state feedback stabilization problem and the second is for fuzzy static output feedback stabilization problem. The first control law gives results more relaxed. However, in realworld control problems, the states may not be completely accessible. In such situations, one needs to resort to output feedback design methods. Fuzzy static output feedback control is the most desirable since it can be implemented easily with low cost. Nevertheless, the fuzzy static output feedback stabilization problem of TS fuzzy systems is rarely investigated because it is quite hard theoretically for TS fuzzy systems but useful and very important in practice. 

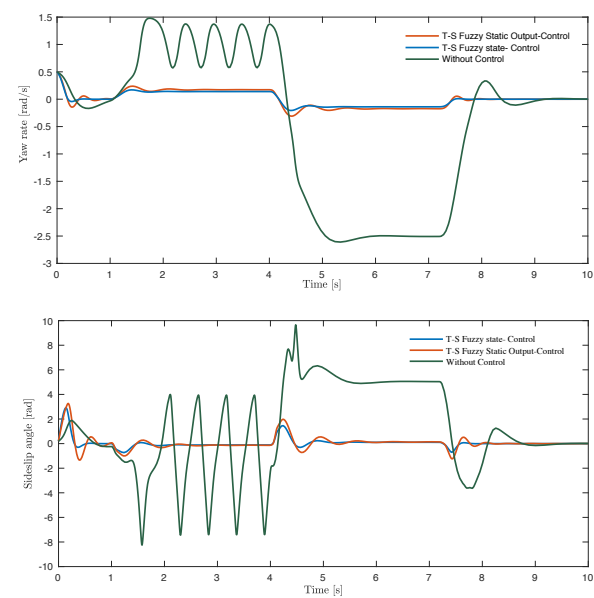

Figure 1. Comparison between controlled system

states (state \& static output- feedback controls) and the uncontrolled
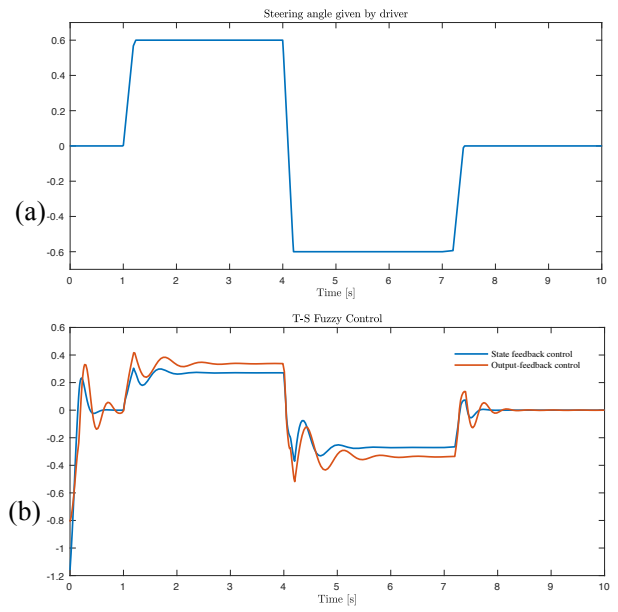

Figure 2. (a) Steering angle given by the driver, (b) Comparison between the response of the two types of control

\section{Keywords:}

Fuzzy state; vehicle lateral dynamics; reconstructed state feedback; output feedback

\section{References}

[1] N. El Youssfi, M. Oudghiri, A. Aitouche \& R. El Bachtiri, "Fuzzy Sliding-Mode Observer for Lateral Dynamics of Vehicles with Consideration of Roll Motion", In $26^{\text {th }}$ Mediterranean Conference on Control and Automation (MED), IEEE, pp. 861-866, 2018.

[2] C. Fang, Y. Liu, S. Kau, L. Hong, C. Lee, "A new LMI-based approach to relaxed quadratic stabilization of TS fuzzy control systems", IEEE Transactions on fuzzy systems, vol. 14, pp. 386-397, 2006.

[3] H. B. Pacejka, “Tyre and Vehicle Dynamics”, Oxford: Butterworth-Heinemann, 2006, p. 657.

\section{Acknowledgement}

The committee of the International Meeting on Advanced Technologies in Energy and Electrical Engineering would like to thank Hamad Bin Khalifa University Press for publishing and hosting the accepted abstracts in QScience.com. 